\title{
ANALYSIS OF CONTROLLED MOLECULAR DYNAMIC FLOW IN A CHANNEL WITH NON-EQUAL INLET AND OUTLET CROSS-SECTIONAL AREAS
}

\author{
Hamed R. Najafi, S.M. Hossein Karimian \\ Department of Aerospace Engineering, Amirkabir University of Technology, Tehran, Iran \\ e-mail: hamedramezani@aut.ac.ir; hkarim@aut.ac.ir
}

\begin{abstract}
Thermo-fluid properties are required for numerical modeling of nano/micro devices. These properties are mostly obtained from the results of molecular dynamics (MD) simulations. Therefore, efforts have been made to develop methods for numerical evaluation of fluid properties such as pressure and velocity. One of the main challenges faced by numerical simulations is to simulate steady molecular flow in channels with non-equal inlet and outlet boundaries. Currently, periodic boundary conditions at the inlet and outlet boundaries are an inevitable condition in many steady flow molecular dynamics simulations. As a result, a nano-channel with different cross sectional areas at the inlet and outlet could not be simulated easily. Here, a method is presented to generate and control steady molecular flow in a nano-channel with different cross sectional areas at the inlet and outlet. The presented method has been applied to a converging-diverging channel, and its performance has been studied through qualitative and quantitative representation of flow properties.
\end{abstract}

Keywords: molecular dynamics, nano-channel, steady flow, non-equal inlet and outlet, pressure

\section{Introduction}

Flow behavior at micro and nano scales has been the subject of interest in the recent years. As a typical reduced-size fluid flow system, flow in a nanochannel can demonstrate different aspects of nanofluid systems. Therefore, several investigations have been focused on the simulation of nanochannel flows (Mi and Chwang, 2003).

Molecular Dynamics (MD) is a deterministic method to calculate the position of molecules and their dynamic properties. Different potential functions have been introduced to represent molecular forces in MD simulations (see for example Leach, 1999; Sadus, 2002). Extracting macroscopic properties such as velocity, temperature and pressure from microscopic data has also been a challenging issue (Allen and Tildesley, 1987; Karniadakis et al., 2002; Karimian et al., 2011).

In earlier studies, the characterization of domain boundaries was not of the primary importance and thus they were treated as simple as possible. In most of the cases, therefore, periodic boundary conditions were used in MD simulations (Stillinger and Rahman, 1974; Travis and Evans, 1977; Koplik et al., 1989; Travis and Gubbins, 2000; Nagayama and Cheng, 2004). However, due to the rapid advancement of computational facilities in the recent years, new problems with more complex boundaries have become a subject of MD simulations. As a result, implementation of realistic boundary conditions in MD simulation is now the center of attention, especially in the field of fluid mechanics (Sun and Ebner, 1992; Huang et al., 2004; Hanasaki and Nakatani, 2006; Huang et al., 2006).

One of the MD applications today that can practically be very beneficial is to present a method for simulation of a specific known or predefined flow in a nanochannel with non-equal 
inlet and outlet cross-sectional areas. Different approaches can be found in the literature to create flow fields in a nanochannel. The most simple and straightforward method is to implement an external force or acceleration to move molecules in a specified direction (Koplik et al., 1988; Fan et al., 2002; Ziarani and Mohamad, 2005; Sofos et al., 2009; Kamali and Kharazmi, 2011). Other than this, some has used motion of a piston or plate upstream of the inlet to create flow in nanotubes (Huang et al., 2004, 2006; Hanasaki and Nakatani, 2006). Implementation of flow gradients such as the temperature gradient can also be used to create nanoflow (Han, 2008; Liu and Li, 2010; Darbandi et al., 2011; Bao et al., 2015). In addition to these methods, in another method, wall motion has been used to create molecular flow in nanochannels (Zhang et al., 2009; Kim et al., 2010).

Implementation of a periodic boundary condition at the inlet and outlet is inevitable in all the above-mentioned methods except for the second one in which a piston upstream of the inlet is used to move the molecules. However, the disadvantage of this approach is that the solution is limited to the period at which the piston moves in its stroke. Also, since additional molecules are to be taken into account in stroke space upstream of the inlet, extra computational efforts are required in this method.

While all of the above-mentioned methods achieve the goal of creating molecular flow in nanochannels, they fail to model steady flow in a channel with different inlet and outlet cross-sectional areas since they use a periodic boundary condition at the inlet and outlet. Also, none of the above methods offers a proper approach to produce specific known or predefined flow in nanochannels. Therefore, the main objective of present study is to investigate flow behavior in a channel of non-equal inlet and outlet cross-sectional areas with controllable flow. In the following sections, we first introduce the calculation method used here to evaluate macroscopic properties in nanoflow. Then we will discuss details about the nanoflow controlled by a temperature gradient. Finally, we introduce a method to solve molecular dynamic flow in a non-equal inlet and outlet cross-sectional-area channel controlled by the temperature gradient.

Finally, a short review on boundary conditions is required since they are implemented on the nanotube wall. The most common option is to model the walls by two rows of molecules. The wall molecules are allowed to oscillate about their initial positions where they are fixed. This is set up either by assigning heavy weights to the wall molecules (Koplik et al., 1988), rescaling the velocity of wall molecules to their initial values (Huang et al., 2004), or using fictitious springs (Fan et al., 2002; Huang et al., 2006; Sofos et al., 2009; Branam and Micci, 2009; Kamali and Kharazmi, 2011). Meanwhile, some studies can be found where walls are modeled differently. An example of such investigations is the study of Ziarani and Mohamad (2005), where the walls were modeled as reflective. The investigation of wall boundary conditions is not the subject of the present study, and the interested scientists are encouraged to refer to the literature for more details. As will be described later, two rows of molecules that are allowed to oscillate about their initial fixed positions represent channel walls.

\section{Pressure and temperature formula in molecular dynamics}

In this paper, the Non-Equilibrium Molecular Dynamics (NEMD) technique is used to simulate flow of Argon. In molecular dynamics, positions of molecules are determined using Newton's second law. Intermolecular forces are calculated using Lennard-Jones 12-6 potential equation (Rapaport, 2004) with a small correction by truncating at the cut-off distance and shifting to eliminate the discontinuity (Leach, 1999)

$$
V\left(r_{i j}\right)= \begin{cases}4 \varepsilon\left[\left(\frac{\sigma}{r_{i j}}\right)^{12}-\left(\frac{\sigma}{r_{i j}}\right)^{6}-\left(\frac{\sigma}{r_{c}}\right)^{12}+\left(\frac{\sigma}{r_{c}}\right)^{6}\right] & \text { for } \quad r_{i j} \leqslant r_{c} \\ 0 & \text { for } \quad r_{i j}>r_{c}\end{cases}
$$


where $r_{i j}$ is the intermolecular distance between atoms $i$ and $j, \varepsilon$ is the energy scale. We choose a cut-off distance of $r_{c}=2.5 \sigma$ herein, which has commonly been used in other studies to simulate Argon flow (Koplik et al., 1989; Priezjev, 2007; Sofos et al., 2009; Bao et al., 2015). $\sigma$ is the finite distance at which the intermolecular potential is zero, and it is equal to molecular diameter in the hard sphere model. In this paper, wall and fluid molecules are formed from the same material. Therefore, all interaction between wall molecules, fluid molecules or wall-fluid molecules follows the above equation with Argon constants.

In a macroscopic analysis, pressure is calculated solely based on the virial equation of state. In a microscopic system however, pressure is calculated from the virial equation of state on all atoms plus the summation of intermolecular forces multiplied by the corresponding distances between them. For pair-additive force fields, the fluid pressure $P$ can be estimated through the virial equation of state given below (Allen and Tildesly, 1987)

$$
P=\frac{N k_{B} T}{V}+\frac{1}{3 V} \sum_{i=1}^{N} \sum_{j>i}^{N} r_{i j} f_{i j}
$$

where $N$ is the number of molecules in the computational domain, $k_{B}$ is Boltzmann's constant, and $V$ and $T$ are volume and temperature of the computational domain, respectively. $r_{i j}$ is the distance between two interacting molecules $i$ and $j$, and $f_{i j}$ is the force of molecule $j$ acting on molecule $i$. The first term in Eq. (2.2) is called the kinetic term and contains temperature of the computational domain. In a microscopic view, temperature is defined as

$$
T=\frac{m}{3 N k_{B}} \sum_{n=1}^{N} \sum_{i=1}^{3}\left(V_{n i}-\bar{V}_{i}\right)^{2}
$$

where $n$ denotes the molecule number in the domain, $i=1,2,3$ denotes the $x, y$ and $z$ components of the atomic velocity $\mathbf{V}_{n}, \bar{V}_{i}$ is the $i$-th component of the mean flow velocity $\overline{\mathbf{V}}$, and $m$ is the atom mass. Mean velocity can be obtained from each of the several averaging methods like SAM (Tysanner and Garcia, 2004), CAM (Tysanner and Garcia, 2005) or SMC (Karimian et al., 2011) which reduces statistical errors in its calculation. In the present study, the SMC method is used to calculate mean velocity. The velocity difference within parentheses that represents instantaneous velocity due to thermal fluctuations is called virial velocity.

The pressure calculation method defined by Eqs. (2.2) and (2.3) has been implemented in an in-house MD code developed in FORTRAN to extract pressure values from the results of MD simulations. This code has been used in authors' previous studies, where its accuracy in calculation of macroscopic properties was demonstrated (Karimian et al., 2011; Karimian and Namvar, 2012; Namvar and Karimian, 2012; Karimian and Izadi, 2013; Hasheminasab and Karimian, 2015; Najafi and Karimian, 2016).

It should be noted that in regions near the boundary, all of the molecules including those outside domain boundary should be taken into account within their cut-off region. This is crucial for correct calculation of pressure (Najafi and Karimian, 2016).

\section{Controlling flow with the temperature gradient in a channel with non-equal inlet and outlet cross-sectional area}

In (Najafi and Karimian, 2016), it was shown that uniform flow of molecules can be created by enforcing two macroscopic properties such as temperature and mean flow velocity at a control point; e.g. at a bin. Najafi and Karimian (2016) also showed that flow of molecules with a gradient of flow properties, such as temperature, can be created if one flow property is defined on an additional control point as well. In fact, they were able to set up a desired periodic flow. In 
the present study, we would like to apply the approach of Najafi and Karimian to set up a desired flow field in a channel with non-equal inlet and outlet cross-sectional areas. To investigate this idea, the following test case is defined.

Geometry of the nano channel considered here is a non-symmetric converging-diverging channel with length of $126.525 \AA$, inlet cross-sectional area of $36.15 \AA \times 36.15 \AA$, outlet cross-sectional area of $29.05 \AA \times 36.15 \AA$, and ratio of throat to inlet cross-sectional areas of 0.6 , as shown in Fig. 1. Seven bins with equal lengths of $18.075 \AA$ are located along the channel. Note that the bin length cannot be very small. In this case, a sufficient number of molecules will not exist in each bin and, therefore, fluctuations will appear in the calculated properties. On the other hand, although smooth results are obtained in larger bins with a moderate number of molecules, they cannot represent local properties accurately. More details about the effect of bin size on sampling and averaging can be found in (Karimian and Izadi, 2013). Wall molecules in bin 1 are arranged in a straight line. From the beginning of bin 2 to the end of bin 7 , the wall molecules are located on a half-sinusoidal profile. The throat of this channel is located at the boundary of bins 5 and 6 that is $90.375 \AA$ from the inlet. The wall profile of bins 6 and 7 is in symmetry with that of bins 5 and 4 . This non-symmetric channel is in fact the first part of a fully symmetric converging-diverging channel with a length of $180.75 \AA$. As mentioned above, the desired flow field can be developed in this channel by enforcing temperature and mean velocity at bin 2 , and temperature at bin 7 .

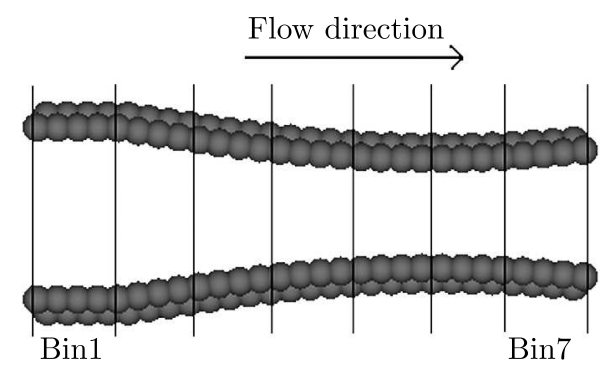

Fig. 1. Non-symmetric channel with 7 bins along the $x$ direction and different inlet and outlet cross-sectional areas

In order to verify our results, we need to know the solution of molecular flow in this channel for the desired values of mean velocity and temperatures at these two bins. For this purpose, we can assume that the above-defined non-symmetric channel is a part of the symmetric converging-diverging channel for which its solution is available. In this case, the solution of the non-symmetric channel would be equal to the solution of this symmetric channel in its first $126.525 \AA$ provided that values of mean velocity and temperatures extracted from the solution of the symmetric converging-diverging channel are enforced at bins 2 and 7 .

Therefore, we first solve molecular flow in a $180.75 \AA$ length symmetric converging-diverging channel, which has the exact wall profile of our test case in its first $126.525 \AA$. A schematic of this channel with its initial configuration of molecules is shown in Fig. 2. This symmetric channel is solved with the periodic boundary condition, and has exactly the same 7 bins of our test case plus 3 extra bins in its $54.225 \AA$ extended part. Obviously, this symmetric converging-diverging channel has an inlet/outlet cross-sectional area of $36.15 \AA \times 36.15 \AA$, and the ratio of throat to inlet cross-sectional areas of 0.6.

This channel contains mono-atomic molecules of Argon gas with an atomic diameter of $\sigma=3.4 \AA$ and energy parameter of $\varepsilon=1.67 \cdot 10^{-21} \mathrm{~J}$. Argon molecules are initially arranged in a FCC lattice form. Solution starts from the initial temperature of $120 \mathrm{~K}$ and zero mean velocity. The periodic boundary condition is applied in the $x$ and $z$ directions. In the $y$ direction, the channel is restricted at each side by two rows of fluid molecules as channel walls. Wall molecules are allowed to oscillate about their initial fixed positions. Temperature of the wall molecules 


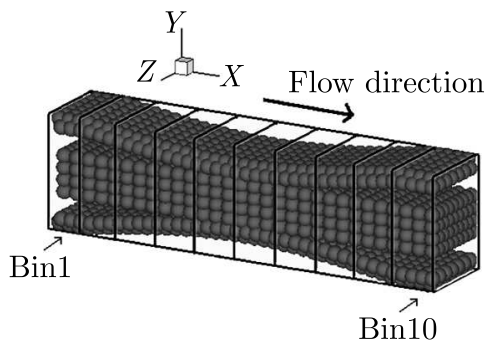

Fig. 2. Schematic of molecule positions in the symmetric converging-diverging channel with 1062 fluid molecules and 1092 wall molecules

is maintained at $120 \mathrm{~K}$ by scaling of the molecule velocities. This channel contains 1062 flow molecules $(N)$ and 1092 wall molecules $\left(N_{w}\right)$. As seen in Fig. 2, the flow molecules are initialized over the channel in a rectangular cube. Each wall has 546 molecules, arranged in a FCC lattice form in a half-sinusoidal profile. Thus, molecular densities of the wall and fluid are $2237.79 \mathrm{~kg} / \mathrm{m}^{3}$ and $1108.18 \mathrm{~kg} / \mathrm{m}^{3}$, respectively. Low amplitude oscillations of the wall molecules, managed by a spring stiffness coefficient of $5000 \mathrm{~N} / \mathrm{m}$, decrease the possibility of molecule escape from the domain boundaries.

The equation of motion is integrated over time with time steps of $10^{-16} \mathrm{~s}$ using the Verlet scheme (Verlet, 1967). Note that the time step value should be chosen appropriately. Very small time steps increase computational cost and solution time, and large time steps may cause solution divergence. The solution domain is divided into 10 equal bins. The solution is preceded for 15000000 time steps to reach equilibrium.

During the solution, temperature of $300 \mathrm{~K}$ and mean velocity of $20 \mathrm{~m} / \mathrm{s}$ are continuously enforced in the second bin. At each time step, mean velocity in this bin is modified by adding the difference between desired and calculated mean velocities to the velocity of each molecule inside the bin. In a similar fashion, temperature in the bin is updated by rescaling the virial velocity of molecules at each time step. The SMC averaging method (Karimian et al., 2011) is applied to calculate pressure and velocity at each time step using Eq. (2.2). Pressure, mean flow velocity, molecular density and temperature variations along the channel are shown in Fig. 3. Convergence rates of velocity and temperature in the bins are shown in Fig. 4.

(a)

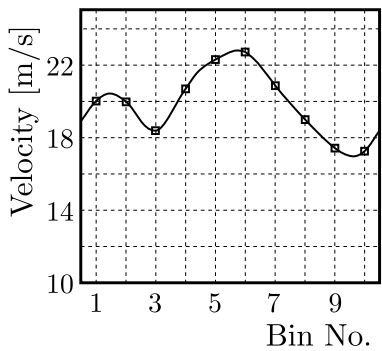

(b)

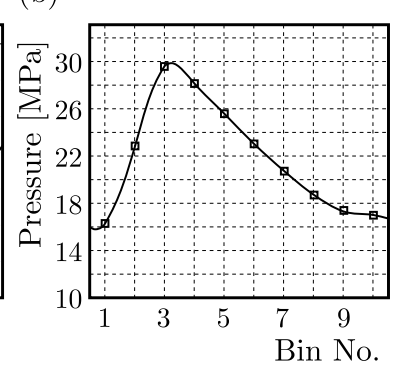

(c)

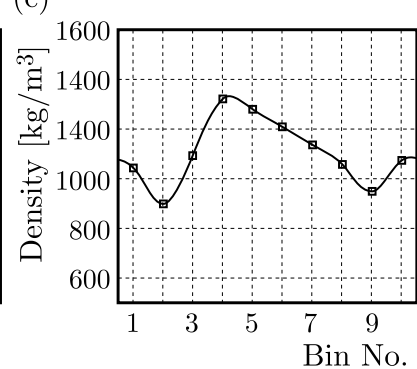

(d)

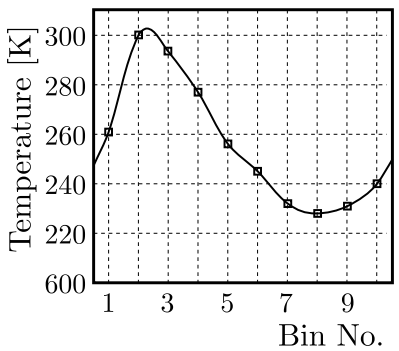

Fig. 3. Variations of flow properties along the symmetric converging-diverging channel generated by enforcement of temperature and mean velocity in bin 2, (a) velocity, (b) pressure and (c) molecular density (d) temperature

It can be seen in Fig. 3a that the mean flow velocity increases through the channel from the inlet to throat and, after that, decreases towards the end of the channel. The throat of this channel is located between bins 5 and 6 . Velocity enforcement at bin 2 results in molecules to be driven towards the flow direction and leave the bin. Therefore, this bin would have the least molecular density. Higher concentration of molecules in bin 3 leads to lower velocities and higher pressures at this bin. 
(a)

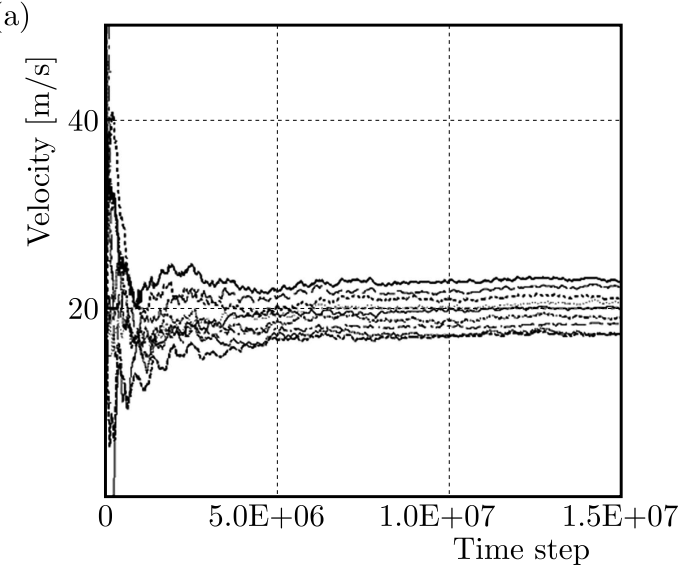

(b)

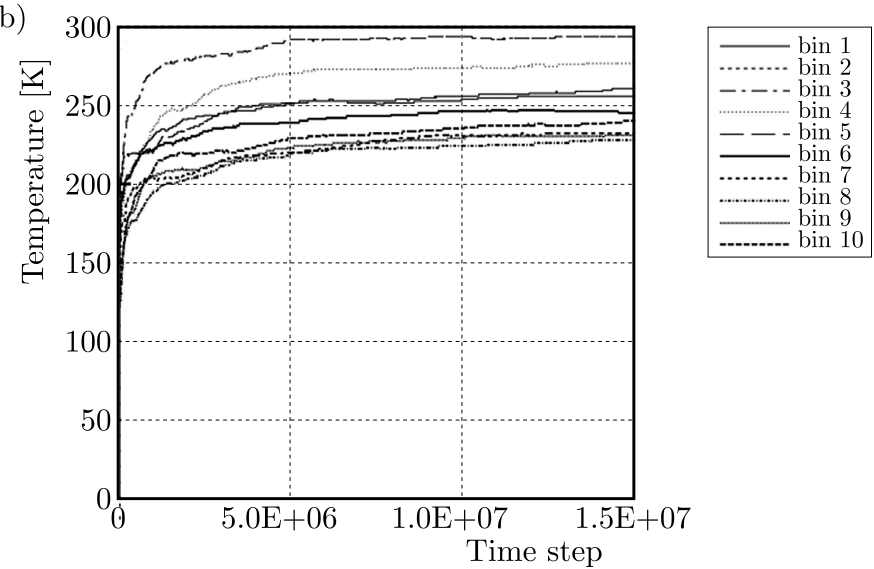

Fig. 4. Convergence rates of: (a) velocity and (b) temperature, along the symmetric converging-diverging channel generated by enforcement of temperature and mean velocity in bin 2

As shown in Fig. 3c, the highest value of molecular density occurs in bin 4 before the throat. Temperature decreases from the control bin through the end of the channel, and the minimum temperature occurs in bin 8 . The temperature setting at the control bin influences upstream bins at lower flow velocities. So the temperature rise could be observed in bins 1, 9 and 10. According to Eq. (2.2), the pressure increases with temperature only through the first term while the effect of molecular density appears in both terms, giving the temperature the dominant role in determining the pressure value and its variation. Therefore, the highest pressure has occurred at bin 3 near the highest temperature bin, as can be seen in Fig. 3b. It should be noted that the flow energy decreases through the channel by restricting and scaling temperature of wall molecules to a fixed value.

As mentioned before, these results will be used to verify the solution of our test case with non-equal inlet and outlet cross-sectional areas, defined in Fig. 1. This non-symmetric channel includes 7 bins. The equation of motion is integrated over time with time steps of $\Delta t=10^{-16}$ second using the Verlet scheme. The solution proceeds for 15000000 time steps to reach equilibrium. Similar to the symmetric channel flow, temperature of $300 \mathrm{~K}$ and mean velocity of $20 \mathrm{~m} / \mathrm{s}$ are continuously enforced in the second bin. In addition, temperature of $234 \mathrm{~K}$ is enforced at the outlet of the channel in bin 7. This temperature has been extracted from the solution of the symmetric converging-diverging channel introduced in Fig. 3. There are 756 wall molecules and 803 fluid molecules between bins 1 to 7 which are equal to those in the same bins of the symmetric converging-diverging channel at the equilibrium. The number of molecules is not changed during the solution. This means that for each molecule leaving the end of the channel, either it should return back into the channel at the inlet or one molecule should be inserted into the domain at the inlet. Insertion of molecules into the domain creates drastic intermolecular forces and change the internal energy in the domain. Such instantaneous forces sometimes cause unexpected disorder in the motion of molecules, which normally results in divergence of the solution. Some methods have been presented to perform the insertion process with minimum changes in the domain internal energy (Sun and Ebner, 1992; Li et al., 1998). In those methods however, inserted molecules may overlap with other molecules and, therefore, due to high internal forces between them, one or both of them may be ejected out of the domain.

Due to difficulties associated with insertion of molecules at the inlet, it has been decided to use a periodic boundary condition at the inlet and outlet of the channel. In this case, every molecule that leaves the solution domain at the outlet will be returned back into the solution domain at the inlet without any difficulty. However, the problem is that cross-sectional areas of the inlet and outlet are not equal to each other. To resolve this problem, the molecules leaving 
the outlet of the non-symmetric channel will be sent into an adaption zone which expands the flow cross-sectional area from the outlet of the non-symmetric channel to an area equal to the inlet cross-sectional area of the channel, i.e. from area of $29.05 \AA \times 36.15 \AA$, to an area of $36.15 \AA \times 36.15 \AA$. Note that the periodic boundary condition is applied between the outlet of the non-symmetric channel and the inlet of the adaption zone, and also between the outlet of the adaption zone and the inlet of the non-symmetric channel. Different profiles can be designed for the wall geometry in this zone; this will be discussed later in this Section. But we know that the best option is to select a wall profile similar to the part of the symmetric converging-diverging channel that has been cut off from it, i.e. a part of the channel with bins 8 to 10, as shown in Fig. 5. This adaption zone, denoted by 1st, is filled with 259 Argon molecules to bring the number of mono-atomic Argon molecules to 1062 in the solution domain. Results obtained from the numerical solution of molecular flow in the non-symmetric channel are compared with the results of the symmetric converging-diverging channel in Fig. 6.

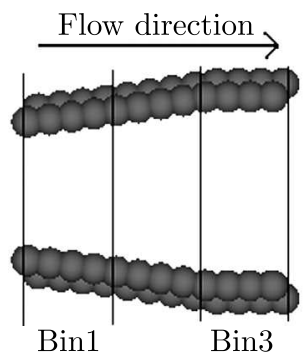

Fig. 5. 1st adaptation zone with 3 bins along the $x$ direction
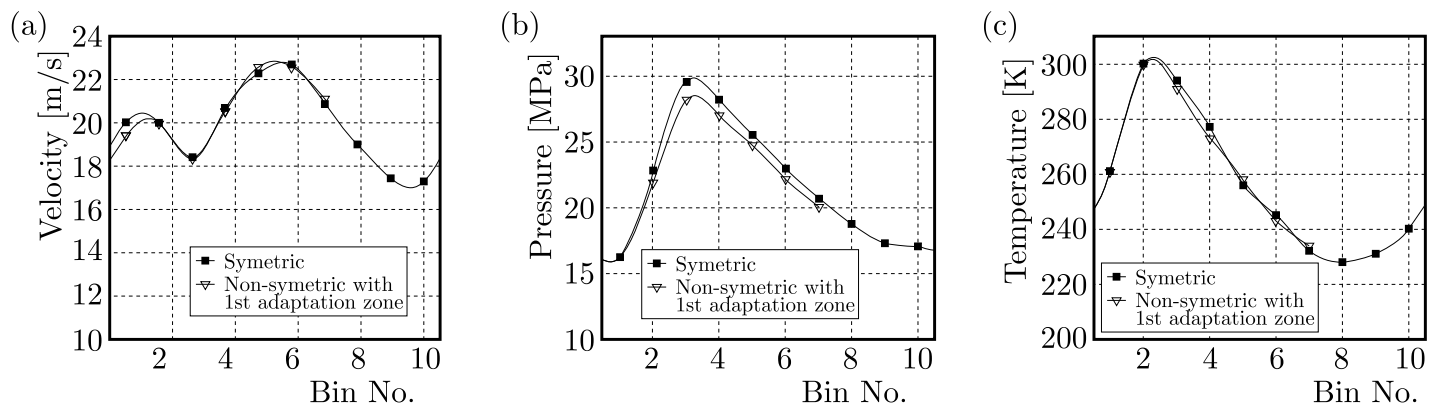

Fig. 6. Variations of flow properties along the non-symmetric channel with temperature of $300 \mathrm{~K}$ and mean velocity of $20 \mathrm{~m} / \mathrm{s}$ at bin 2 and temperature of $234 \mathrm{~K}$ at bin 7 in comparison to those of the symmetric channel, (a) mean flow velocity, (b) pressure, and (c) temperature

As shown in Fig. 6, the agreement between results obtained from the solutions of symmetric and non-symmetric channels is excellent, except for the pressure variation. Note that since temperature of bin 7 is set up to $234 \mathrm{~K}$ by rescaling of the Virial velocity of molecules in this bin at each time step, the arrangement of molecules in this bin will be changed in comparison to that of the symmetric converging-diverging channel. Due to this rearrangement of molecules in bin 7 , temperature variation is slightly underestimated. As noted before, temperature has the main role in the determination of pressure. Therefore, rearrangement of molecules in bin 7 has affected pressure through both terms of Eq. (2.2). As a result, a small difference between the results of pressure depicted in Fig. $6 \mathrm{~b}$ is inevitable. Therefore, we have been able to generate specific flow in a channel with non-equal inlet and outlet cross-sectional areas by enforcement of temperature and mean velocity at one bin, e.g. bin 2 here, and temperature at another bin, e.g. bin 7 here.

In order to produce extra validation data for comparison purposes, molecular flow of Argon in the symmetric converging-diverging channel of Fig. 2 has been solved for two additional 
conditions at bin 2: (a) temperature of $300 \mathrm{~K}$ and mean velocity of $50 \mathrm{~m} / \mathrm{s}$, and (b) temperature of $300 \mathrm{~K}$ and mean velocity of $100 \mathrm{~m} / \mathrm{s}$. Other settings of these two numerical solutions are exactly the same as those of the first solution with temperature of $300 \mathrm{~K}$ and mean velocity of $20 \mathrm{~m} / \mathrm{s}$. Solutions of cases (a) and (b) indicate that temperature of bin 7 in these two cases reaches $263 \mathrm{~K}$ and $279 \mathrm{~K}$, respectively. Now, once again molecular flow is solved in the non-symmetric channel, having enforced temperature and mean velocity at bin 2, and extracted temperature at bin 7 . All other settings of the solution are as before. Comparison of pressure, mean flow velocity, molecular density and temperature variations along the non-symmetric channel with those of the symmetric converging-diverging channel are shown in Fig. 7.

(a)

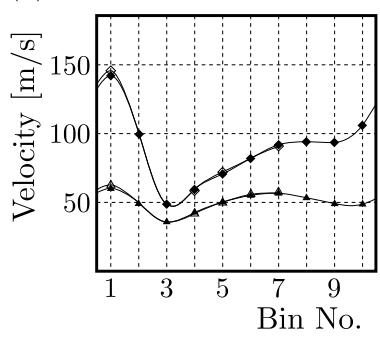

(b)

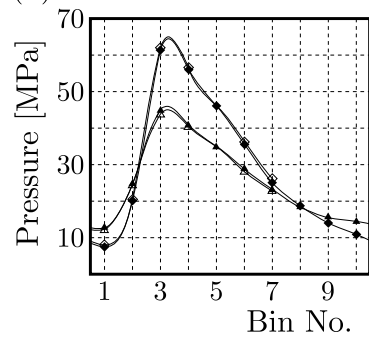

(c)

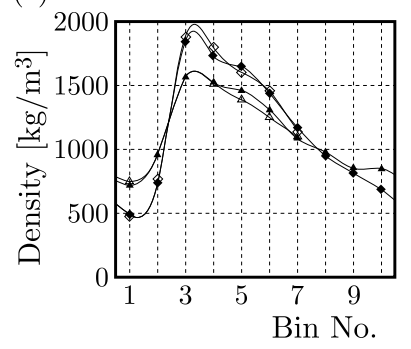

(d)

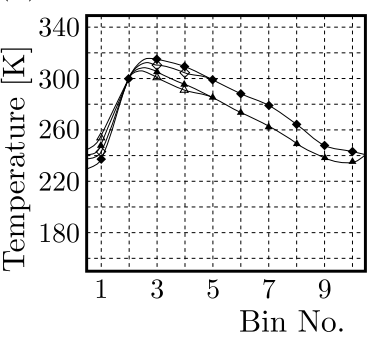

ـ Symmetric $(50 \mathrm{~m} / \mathrm{s})$

- Symmetric $(100 \mathrm{~m} / \mathrm{s})$

$\triangle$ Non-symmetric with 1 st adaptation zone $(50 \mathrm{~m} / \mathrm{s}) \diamond$ Non-symmetric with 1 st adaptation zone $(100 \mathrm{~m} / \mathrm{s})$

Fig. 7. Comparison of velocity, pressure, molecular density and temperature along the non-symmetric channel with those of the symmetric converging-diverging channel for two cases of (a) temperature of $300 \mathrm{~K}$ and mean velocities of $50 \mathrm{~m} / \mathrm{s}$ at bin 2 and temperatures of $263 \mathrm{~K}$ at bin 7 , and (b) temperature of $300 \mathrm{~K}$ and mean velocities of $100 \mathrm{~m} / \mathrm{s}$ at bin 2 and temperatures of $279 \mathrm{~K}$ at bin 7

Variation of the mean flow velocity along the channel shows a slightly different trend at $100 \mathrm{~m} / \mathrm{s}$ in comparison to $50 \mathrm{~m} / \mathrm{s}$ case as seen in Fig. 7a. Due to the accumulation of molecules in the throat region, the mean velocity of molecules decreases in the throat upstream for both cases. Downstream the throat however, molecules gain velocity as a result of less concentration in this region. It could be seen in Fig. 7b that pressure increases with molecular density upstream the throat. In this region, higher temperature has also caused the pressure to increase, according to Eq. (2.2). The agreement between the results of the symmetric and non-symmetric channels is more realized at higher mean flow velocities. Therefore, it is clear that the presented method is more accurate at higher velocities of mean flow.

Again, comparison of the results in Fig. 7 proved that the presented approach has been able to generate specific flow in the channel with non-equal inlet and outlet cross-sectional areas by enforcement of temperature and mean velocity at one bin, and temperature at another bin if a proper adaption zone is used. In order to generalize this approach, it is needed to investigate the effect of adaption-zone geometry on the results. It is significant to demonstrate that the presented approach is mainly independent of the shape of the adaption zone. The adaption zone is characterized by its length, its wall slopes at the inlet and outlet, and cross-sectional areas at the inlet and outlet. For any channel, the latter one is fixed, i.e. from inlet of $29.05 \AA \times 36.15 \AA$ to outlet of $36.15 \AA \times 36.15 \AA$. Therefore, we define test cases to investigate the effect of other two parameters, i.e. length and slope of the adaption zone.

First, two different adaption zones shown in Fig. 8 are investigated. Test case (b) where mean flow velocity of $100 \mathrm{~m} / \mathrm{s}$ is enforced at bin 2 is solved with these adaption zones. The adaptation zones proposed in Figs. $8 \mathrm{a}$ and $8 \mathrm{~b}$ as $2 \mathrm{nd}$ and $3 \mathrm{rd}$ geometries include three and two bins, respectively; i.e. equal to $54.225 \AA$ and $36.15 \AA$ in length, respectively. In both cases, the slope of the wall profile is 0.196 in the first bin, and is zero in the other bins.

The number of fluid molecules in the 2nd and 3rd adaptation zones of Figs. 8a and Fig. 8b are equal to 259 and 153, respectively. The number of molecules in the geometries has been selected 
(a)

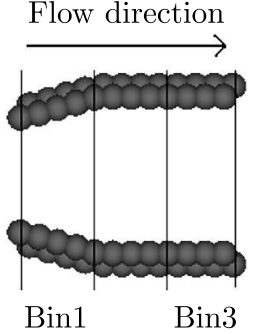

(b)

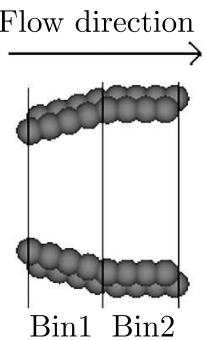

Fig. 8. Modified adaptation zones: (a) 2nd geometry (b) 3rd geometry

based on the average density of molecules in the non-symmetric channel. Therefore, the added number of molecules is proportional to the adaptation zone volume. The results obtained from these two solutions are compared with those obtained from the symmetric converging-diverging channel and from the non-symmetric channel with the 1st adaption zone in Fig. 9.

(a)

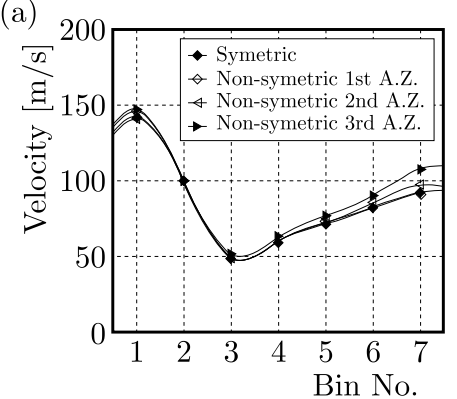

(b)

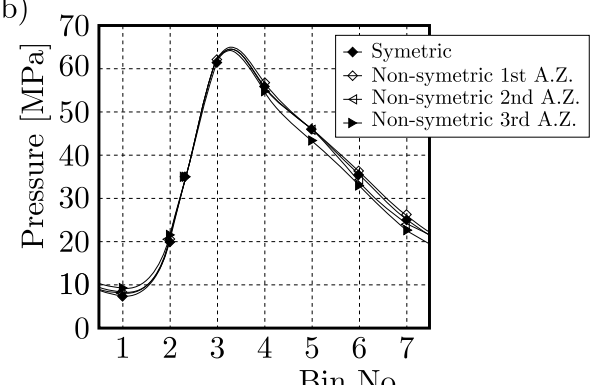

(c)

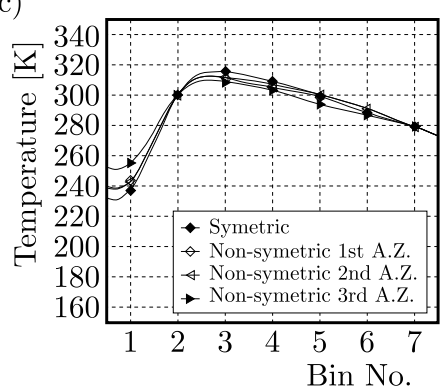

Fig. 9. Comparison of velocity, pressure and temperature variations of flow variables along the non-symmetric channel generated by temperature of $300 \mathrm{~K}$ and mean velocity of $100 \mathrm{~m} / \mathrm{s}$ at bin 2 and temperatures of $279 \mathrm{~K}$ at bin 7 with 1st, 2nd, and 3rd adaption zones, with those of the symmetric channel

In comparison to the results of the symmetric channel and those obtained using the 1st adaption zone, both adaption zones have predicted flow properties very well, however, between the 2 nd and 3 rd adaptation zones, the 2nd adaption zone leads to a better estimation of flow properties. We believe that this is due to the facts that 1) the slope of the 2 nd adaption zone from the inlet to outlet is smoother than that of the 3rd adaption zone, and 2) length of the 2nd adaption zone is longer. But we would like to have an adaption zone with small length in order to minimize computational cost. For this purpose, two additional adaption zones denoted by fourth (4th) and fifth (5th), with $36.15 \AA$ in length are defined. Lengths of both adaption zones are equal to the 3rd adaption zone. The 4th adaption zone shown in Fig. 10a has a smoother wall slope. From the beginning of the first bin up to the middle of the second bin, the wall slope is constant and equal to 0.13, i.e. with an angle of $7.4^{\circ}$. From the middle of the second bin, the slope of the wall is set equal to zero. The 5 th adaption zone shown in Fig. 10b will be introduced after analysis of the results obtained from the 4th adaption zone. The number of fluid molecules in both adaptation zones is equal to 259 .

Having solved molecular flow in the non-symmetric channel using the 4th adaption zone with temperature of $300 \mathrm{~K}$ and mean velocity of $100 \mathrm{~m} / \mathrm{s}$ at bin 2 and temperatures of $279 \mathrm{~K}$ at bin 7 , its results are compared with the results of the symmetric channel and those of the non-symmetric channel with the 2nd and 3rd adaption zones in Fig. 11. Having compared with the results of the symmetric channel, results of Fig. 11 show that using the 4th adaptation zone, the flow properties are estimated better than when the 3rd adaptation zone is used. We believe that this is due to the fact that the wall of the 4 th adaption zone has a lower wall slope. This once again proves that the adaption zone with smoother wall profiles are preferred. Note that 
(a)

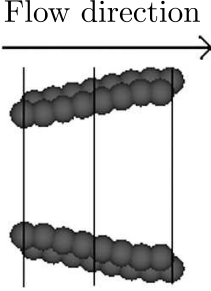

Bin1 Bin2 (b)

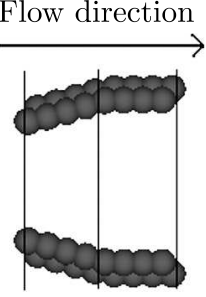

Bin1 Bin2

Fig. 10. (a) 4th adaptation zone (b) 5th adaptation zone
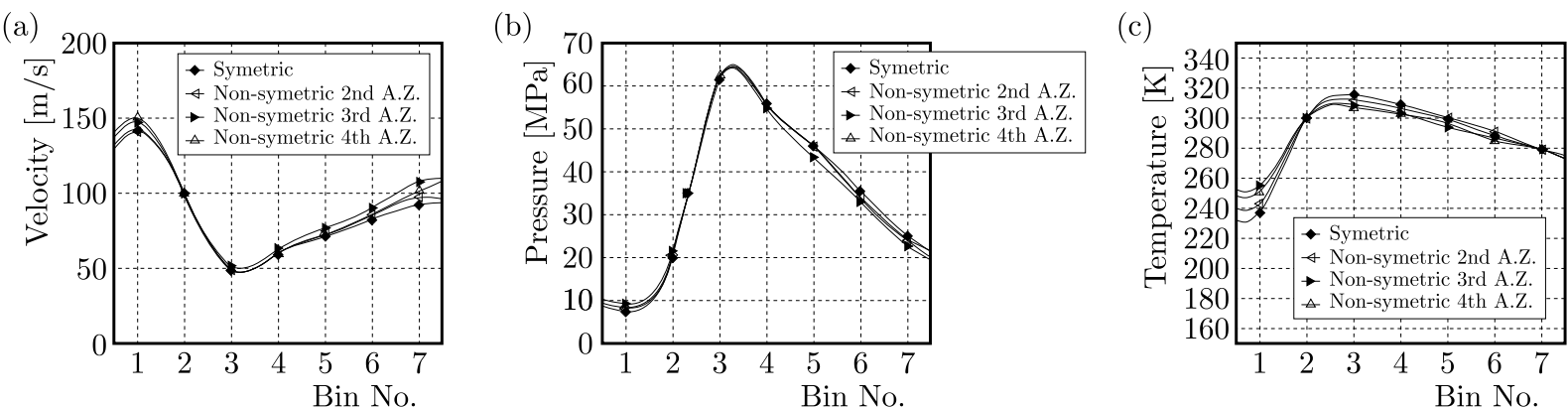

Fig. 11. Comparison of velocity, pressure and temperature along the non-symmetric channel generated by temperature of $300 \mathrm{~K}$ and mean velocity of $100 \mathrm{~m} / \mathrm{s}$ at bin 2 and temperatures of $279 \mathrm{~K}$ at bin 7 with 2nd, 3rd and 4th adaption zones, with those of the symmetric channel

based on Fig. 11, although the results obtained using the 4th adaption zone do not give a better estimation of flow properties in comparison to the results obtained using the 2nd adaptation zone, it costs computationally less since it is shorter in length.

Careful inspection of the 4th adaption zone reveals that the slope of this adaptation zone at its inlet is very close to the slope of the non-symmetric channel at its outlet. Therefore, to achieve more correct results, it is necessary to match the inlet slope of the adaptation zone with the outlet slope of the non-symmetric channel.

To account for these requirements, it is proposed to generate the wall profile of the adaption zone with a 3rd degree (cubic) polynomial which will have an inlet slope equal to the outlet slope of the non-symmetric channel and an outlet slope equal to the inlet slope of the non-symmetric channel. Two other constants of the cubic polynomial are determined by the definition of the beginning and the end coordinates of the adaption zone. This profile that has length equal to that of the 4th adaption zone (2 bins) is shown in Fig. 10b. The previous flow simulation is repeated but using the 5th adaption zone. The results of this simulation including variation of velocity, pressure and temperature along the channel are shown in Fig. 12.
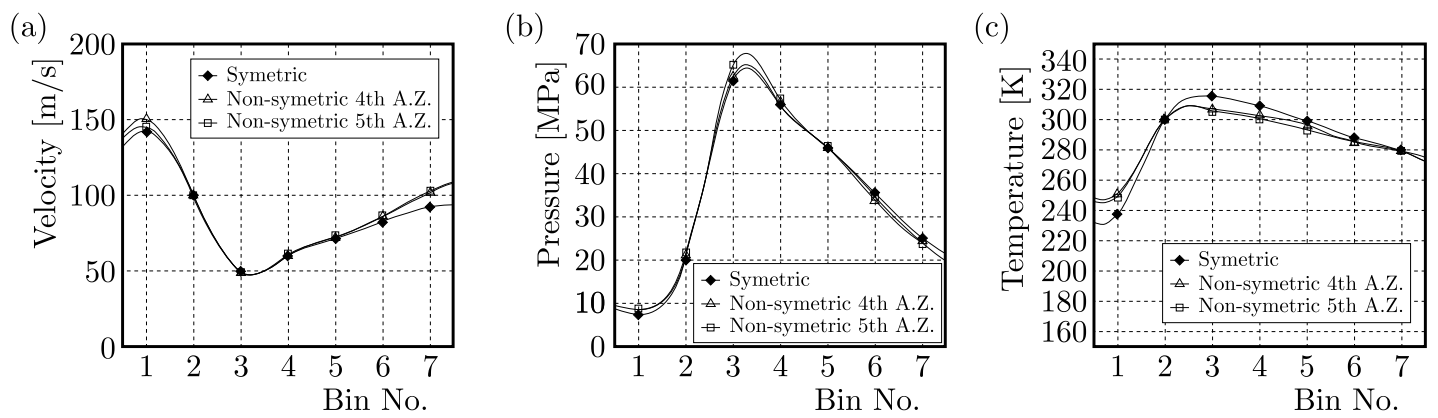

Fig. 12. Comparison between variations of flow variables along the non-symmetric channel generated by temperature of $300 \mathrm{~K}$ and mean velocity of $100 \mathrm{~m} / \mathrm{s}$ at bin 2 and temperatures of $279 \mathrm{~K}$ at bin 7 with 4 th and 5 th adaptation zones, (a) velocity, (b) pressure and (c) temperature 
As seen in Fig. 12, the results obtained using the 4th and 5th adaptation zones are very similar to each other. Especially, accurate results have been achieved at the end of the nozzle.

To show that this method does not depend on the number of molecules, the previous flow simulations are repeated with 200 more and fewer molecules in the symmetric nanochannel and the same trend is observed with the adaptation zones in variation of velocity, pressure and temperature.

\section{Conclusion}

In this paper, it is shown that flow can be generated and controlled in a channel with different inlet and outlet cross sections by setting temperature at two different regions and mean flow velocity at one of these regions. In a channel with non-equal inlet and outlet, periodic boundary conditions cannot be implemented. Therefore, to handle this difficulty, an adaption zone is used to computationally expand the outlet of the channel to a cross sectional area equal to its inlet. This adaption zone should have the following specifications to result in a more accurate flow prediction.

- Length: If its length is too short then the accuracy of estimated flow properties along the channel with variable cross-sectional area decreases. On the other hand, if the length of the channel is too long, then the cost of computation will raise. In the non-symmetric converging-diverging channel, for instance, the making use of the adaptation zone with length and wall profile similar to the part that has been cut off from it, results in a more accurate estimation of flow properties.

- Slope at junctions: To achieve more accurate results, it is necessary to set the inlet slope of the adaption zone equal to the outlet slope of the channel, and also to set the outlet slope of the adaption zone equal to the inlet slope of the channel.

- Wall profile: A 3rd degree (cubic) polynomial is used for the wall profile of the adaption zone. With this profile, slopes at the inlet and outlet, and also length of the adaption zone can be fixed to the desired values and, therefore, guarantee a smooth wall profile. Note that the slope of the channel inlet (and thus the adaption zone outlet) is preferred to be zero.

\section{References}

1. Allen M.P., Tildesley D.J., 1987, Computer Simulation of Liquids, Oxford, New York

2. BaO F., HuAng Y., Zhang Y., Lin J., 2015, Investigation of pressure-driven gas flows in nanoscale channels using molecular dynamics simulation, Microfluid Nanofluid, 18, 1075-1084

3. Branam R.D., Micci M.M., 2009, Comparison of wall models for the molecular dynamics simulation of microflows, Nanoscale and Microscale Thermophysical Engineering, 13, 1-12

4. Darbandi M., Abbassi H.R., Khaledi Alidusti R., Sabouri M., 2011, Molecular Dynamics Simulation of Nano Channel as Nanopumps, ICNMM, Edmonton, Alberta, Canada

5. FAn X.J., Nhan P.T., NG T.Y, Xu D., 2002, Molecular dynamics simulation of a liquid in a complex nano channel flow, Physics of Fluids, 14, 3, 1146-1153

6. Han M., 2008, Thermally-driven nanoscale pump by molecular dynamics simulation, Journal of Mechanical Science and Technology, 22, 157-165

7. Hanasaki I., NakAtani A., 2006, Fluidized piston model for molecular dynamics simulations of hydrodynamics flow, Modelling and Simulation in Materials Science and Engineering, 14, S9-S20 
8. Hasheminasab S.M., Karimian S.M.H., 2015, New indirect method for calculation of flow forces in molecular dynamics simulation, Journal of Molecular Liquids, 206, 183-189

9. Huang C., Choi P.Y.K., Nandakumar K., Kostiuk L.W., 2006, Molecular dynamics simulation of a pressure-driven liquid transport process in a cylindrical nanopore using two self-adjusting plates, Journal of Chemical Physics, 124, 234701

10. Huang C., NAndakumar K., Kwok D.Y., 2004, Non-equilibrium injection flow in a nanometer capillary channel, ICMENS'04, 374-378

11. Kamali R., Kharazmi A., 2011, Molecular dynamics simulation of surface roughness effects on nanoscale flows, International Journal of Thermal Science, 50, 3, 226-232

12. Karimian S.M.H., IzAdi S., 2013, Bin size determination for the measurement of mean flow velocity in molecular dynamics simulation, International Journal for Numerical Methods in Fluids, 71, 7, 930-938

13. Karimian S.M.H., Izadi S., Barati Farimani A., 2011, A study on the measurement of mean velocity and its convergence in molecular dynamics simulations, International Journal for Numerical Methods in Fluids, 67, 12, 2130-2140

14. Karimian S.M.H., Namvar S., 2012, Implementation of SMC averaging method in a channeled molecular flow of liquids and gases, Journal of Physics: Conference Series, 362, 01, 2029

15. Karniadakis G.E., Beskok A., Aluru N., 2002, Micro Flows and Nano Flows, Springer, New York, 641-648

16. Kim B.H., Beskok A., CAgin T., 2010, Viscous heating in nanoscale shear driven liquid flows, Microfluid Nanofluid, 9, 31-40

17. Koplik J., Banavar J., Willemsen J., 1988, Molecular dynamics of Poiseuille flow and moving contact lines, Physical Review Letters, 60, 1282-1285

18. Koplik J., Banavar J.R., Willemsen J.F., 1989, Molecular dynamics of fluid flow at solid surfaces, Physics of Fluids A, 1, 781-794

19. Leach A.R., 1999, Molecular Modeling: Principles and Applications, Longman

20. Li J., LiaO D., YIP S., 1998, Coupling continuum to molecular-dynamics simulation: reflecting particle method and the field estimator, Physical Review E, 57, 6, 7259-7267

21. LiU C., Li Z., 2010, Molecular dynamics simulation of composite nanochannels as nanopumps driven by symmetric temperature gradients, Physical Review Letters, 105, 174501

22. Mi X.B., Chwang A. T., 2003, Molecular dynamics simulations of nanochannel flows at low Reynolds numbers, Molecules, 8, 193-206

23. Nagayama G., Cheng P., 2004, Effects of interface wettability on microscale flow by molecular dynamics simulation, International Journal of Heat and Mass Transfer, 47, 501-513

24. NAJAfi H.R., Karimian S.M.H., 2016, Analysis of pressure behavior in a temperature controlled molecular dynamic flow, Journal of Theoretical and Applied Mechanics, 54, 3, 881-892

25. Namvar S., Karimian S.M.H., 2012, Detailed investigation on the effect of wall spring stiffness on velocity profile in molecular dynamics simulation, Journal of Physics: Conference Series, 362, 01, 2039

26. Priezjev N.V., 2007, Effect of surface roughness on rate-dependent slip in simple fluids, Journal of Chemical Physics, 127, 144708

27. Rapaport D.C., 2004, The Art of Molecular Dynamics Simulation, Cambridge University Press

28. Sadus R.J., 2002, Molecular Simulation of Fluids: Theory, Algorithms and Object-Orientation, Elsevier

29. Sofos F., Karakasidas T.E., Liakopoulos A., 2009, Non-equilibrium molecular dynamics investigation of parameters affecting planar nanochannel flows, Contemporary Engineering Sciences, 2, 6, 283-298 
30. Stillinger F.H., Rahman A., 1974, Improved simulation of liquids water by molecular dynamics, Journal of Chemical Physics, 60, 1545-1557

31. Sun M., EBner C., 1992, Molecular-dynamics simulation of compressible fluid flow in two-dimensional channels, Physical Review A, 46, 4813

32. Travis K.P., Evans D.J., 1997, Molecular spin in a fluid undergoing Poiseille flow, Physical Review E, 55, 1566-1572

33. Travis K.P., Gubbins K.E., 2000, Poiseuille flow of Lennard-Jones fluids in narrow slit pores, Journal of Chemical Physics, 112, 1984-1994

34. Tysanner M.W., Garcia A.L., 2004, Measurement bias of fluid velocity in molecular simulations, Journal of Computational Physics, 196, 173-183

35. Tysanner M.W., Garcia A.L., 2005, Non-equilibrium behavior of equilibrium reservoirs in molecular simulations, International Journal of Numerical Methods in Fluids, 2050, 1-12

36. Verlet L., 1967, Computer "experiments" on classical fluids. I. Thermodynamical properties of Lennard-Jones molecules, Physical Review, 159, 98

37. Zhang Z.Q., Zhang H.W., Ye H.F., 2009, Pressure-driven flow in parallel-plate nanochannels, Applied Physics Letters, 95, 154101

38. Ziarani A.S., Mohamad A.A., 2005, A molecular dynamics study of perturbed Poiseulle flow in a nanochannel, Microfluid Nanofluid, 2, 12-20 